\title{
Biallelic inactivation of REV7 is associated with Fanconi anemia
}

\author{
Dominique Bluteau, ${ }^{1,2}$ Julien Masliah-Planchon, ${ }^{1,2}$ Connor Clairmont, ${ }^{3}$ Alix Rousseau, ${ }^{1,2}$ Raphael Ceccaldi, ${ }^{3}$ \\ Catherine Dubois d'Enghien, ${ }^{4}$ Olivier Bluteau, ${ }^{1,2,5}$ Wendy Cuccuini, ${ }^{5}$ Stéphanie Gachet, ${ }^{1,2}$ Régis Peffault de Latour, ${ }^{2,6}$ \\ Thierry Leblanc, ${ }^{7}$ Gérard Socié, ${ }^{2,6}$ André Baruchel, ${ }^{2,7}$ Dominique Stoppa-Lyonnet, ${ }^{4}$ Alan D. D'Andrea, ${ }^{3}$ and Jean Soulier ${ }^{1,2,5}$ \\ ${ }^{1}$ INSERM U944/CNRS UMR7212, Saint-Louis Hospital, Paris, France. ${ }^{2}$ Institute of Hematology (IUH), University Paris Diderot, Sorbonne Paris Cité, Paris, France. ${ }^{3}$ Center for DNA Damage and Repair, \\ Department of Radiation Oncology, Dana-Farber Cancer Institute, Harvard Medical School, Boston, Massachusetts, USA. ${ }^{4}$ Oncogenetic Laboratory, Curie Institute, Paris, France. ${ }^{5}$ Hematology Laboratory \\ Assistance Publique-Hôpitaux de Paris (APHP), and ${ }^{6}$ Hematology/Transplantation APHP, Saint-Louis Hospital, Paris, France. ${ }^{7}$ Department of Pediatric Hematology, Robert Debré Hospital, Paris, France.
}

Fanconi anemia (FA) is a recessive genetic disease characterized by congenital abnormalities, chromosome instability, progressive bone marrow failure (BMF), and a strong predisposition to cancer. Twenty FA genes have been identified, and the FANC proteins they encode cooperate in a common pathway that regulates DNA crosslink repair and replication fork stability. We identified a child with severe BMF who harbored biallelic inactivating mutations of the translesion DNA synthesis (TLS) gene REV7 (also known as MAD2L2), which encodes the mutant REV7 protein REV7-V85E. Patient-derived cells demonstrated an extended FA phenotype, which included increased chromosome breaks and $\mathrm{G}_{2} / \mathrm{M}_{\text {accumulation }}$ upon exposure to DNA crosslinking agents, $\gamma \mathrm{H} 2 \mathrm{AX}$ and 53BP1 foci accumulation, and enhanced p53/p21 activation relative to cells derived from healthy patients. Expression of WT REV7 restored normal cellular and functional phenotypes in the patient's cells, and CRISPR/Cas9 inactivation of REV7 in a non-FA human cell line produced an FA phenotype. Finally, silencing Rev7 in primary hematopoietic cells impaired progenitor function, suggesting that the DNA repair defect underlies the development of BMF in FA. Taken together, our genetic and functional analyses identified REV7 as a previously undescribed FA gene, which we term FANCV.

\section{Introduction}

Fanconi anemia (FA) is the most frequent cause of inherited bone marrow failure (IBMF) syndromes $(1,2)$. Twenty $F A$ genes have been identified, including FANCA, FANCB, FANCC, FANCD1 (also known as BRCA2), FANCD2, FANCE, FANCF, FANCG, FANCI, FANCJ (also known as BRIP1 or BACH1), FANCL, FANCM, FANCN (also known as PALB2), FANCO (also known as RAD51C), FANCP (also known as SLX4), FANCQ (also known as XPF or ERCC4), FANCR (also known as RAD51), FANCS (also known as BRCA1), FANCT (also known as UBE2T), and FANCU (also known as $X R C C 2)(3-5)$. The products of these genes cooperate in a unique FA/BRCA pathway, regulating the response to physiological stress or exposure to genotoxic agents and maintaining genome integrity (3-5). Most FA patients develop a progressive bone marrow failure (BMF) during childhood due to the depletion or impairment of the hematopoietic stem cell (HSC) pool (1, 2, 6-8).

Here, we identified biallelic mutations in REV7 (also known as $M A D 2 L 2)$ in a child with a classic presentation of FA. Interestingly, REV7 has several cellular functions including translesion DNA synthesis (TLS) $(9,10)$, mitotic checkpoint regulation $(11,12)$, and DNA repair pathway choice $(13,14)$. Which if any of these functions of REV7 is required for suppressing the FA cellular and developmental phenotypes is unknown.

Conflict of interest: The authors have declared that no conflict of interest exists. Submitted: April 12, 2016; Accepted: June 16, 2016.

Reference information: J Clin Invest. 2016;126(9):3580-3584. doi:10.1172/JCI88010.

\section{Results and Discussion}

A child with a clinical and cellular FA phenotype and a constitutive REV7 mutation. Patient EGF123, an 8-year-old girl, presented with severe BMF involving all 3 lineages (hemoglobin, $8.0 \mathrm{~g} / \mathrm{dl}$; neutrophil count, $0.43 \times 10^{9} / 1$; and platelets, $\left.10 \times 10^{9} / 1\right)$. She exhibited FA physical signs (short size at less than tenth percentile, microcephaly, and abnormal facial features), a renal tubulopathy, elevated serum $\alpha$-fetoprotein, and a positive mitomycine $\mathrm{C}(\mathrm{MMC})$ chromosome breakage test of blood lymphocytes (15), establishing a definitive diagnosis of FA (Figure $1 \mathrm{~A})$. Monoubiquitination of the FANCD2 protein in the patient cells suggested an abnormality downstream or independent of the FA core complex (Figure 1B) (16). Functional analysis of the patient's skin fibroblasts and EBV-transformed lymphoid cells confirmed the FA phenotype (i.e., a hypersensitivity to the interstrand-crosslinking [ICL] agent MMC), with increased chromosome radials (Figure 1C), arrest at the $G_{2}$ phase of the cell cycle, and growth inhibition (Figure 1, $\mathrm{D}$ and E). Consistent with previous studies with FA cells (7, 17), MMC exposure strongly activated Ser15 phosphorylation of p53 and CDK$N 1 A$ (also known as $p 21$ ) transcriptional induction (Figure 1, F and G).

Sequencing of known FANC genes failed to identify a deleterious nucleotide variant or deletion. Whole exome sequencing (WES) on genomic DNA from the EGF123 proband identified a homozygous $R E V 7$ variant, c. $354 \mathrm{~T}>\mathrm{A}$. Sanger resequencing confirmed the homozygous variant in EFG123 skin fibroblast and EBV-transformed cells (Figure 1H). Rare regions of germline homozygosity (which included the REV7 gene) were observed in this patient, consistent with distant consanguinity (Supplemental 
A

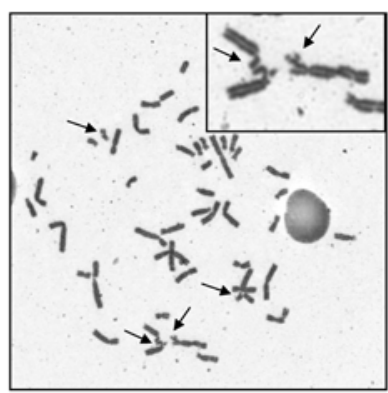

D

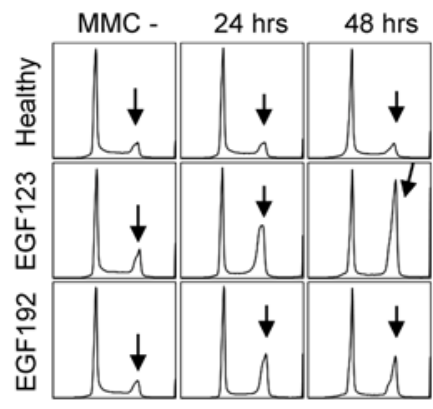

G

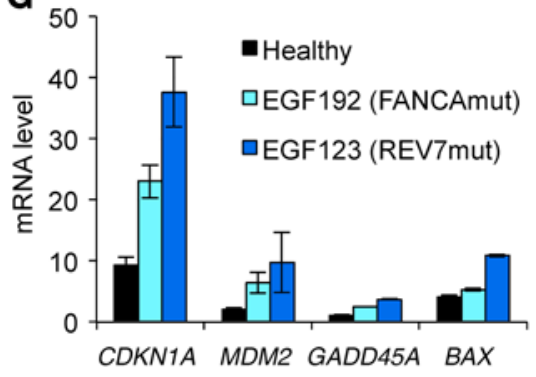

B

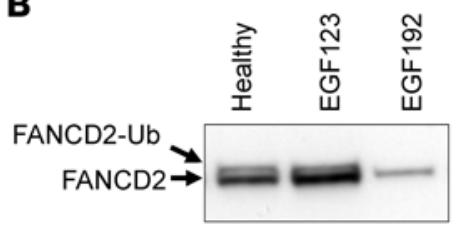

E

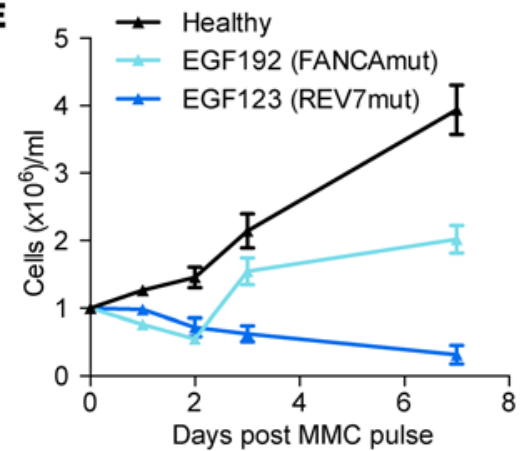

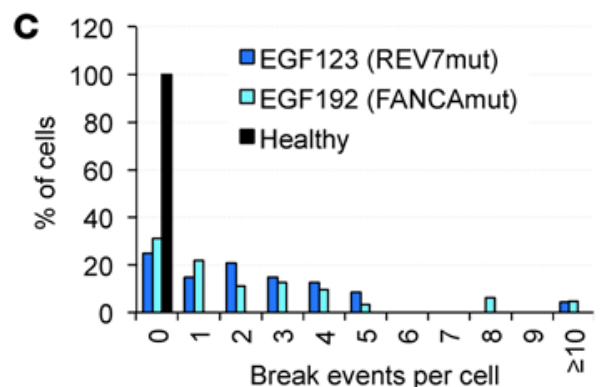

$\mathbf{F}$

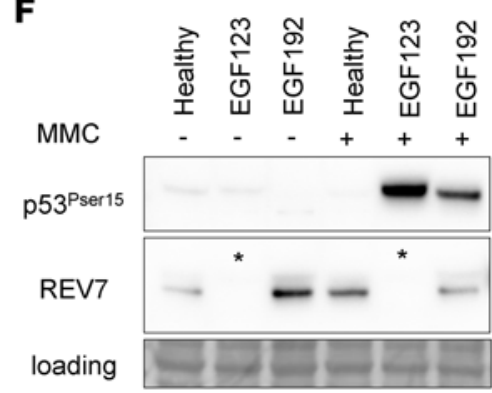

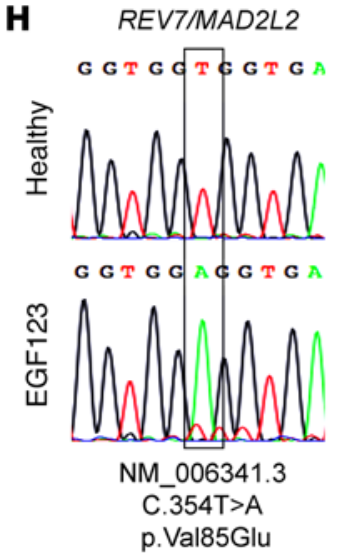

\begin{abstract}
Figure 1. Genetic and cellular phenotype of the REV7-mutated patient EGF123. (A) Metaphase EGF123 lymphoid cells upon MMC exposure; arrows show chromosome breaks. Original magnification, $\times 630$. (B) Immunoblot analysis of FANCD2 monoubiquitination of the REV7mutated (EGF123), FANCA-mutated (EGF192), and healthy fibroblasts. (C) Quantification of the MMC-induced breaks per cell in REV7-mutated (EGF123) and FANCA-mutated (EGF192) EBV-transformed cells and cells of a healthy subject. (D) Cell-cycle analysis after MMC pulse; arrows show the late $S / G_{2}$ arrest. (E) Proliferation curves after MMC pulse. (F) Protein immunoblot analysis before (-) and 24 hours after (+) MMC pulse; asterisks underline the absence of REV7 in EGF123 protein extracts. (C) Transcript expression levels of a set of DNA damage response genes analyzed by quantitative reverse-transcriptase PCR (qRT-PCR) relative to HPRT (primers are indicated in Supplemental Table 1). Experiments shown in panels $\mathbf{E}-\mathbf{H}$ were performed using EBV-transformed cells. $(\mathbf{H})$ Partial REV7 exon5 sequence in gDNA from primary fibroblasts.
\end{abstract}

Figure 1; supplemental material available online with this article; doi:10.1172/JCI88010DS1). The c.354 C>T REV7 is a variant based on a survey of publicly accessible variant databases. The mutation affects an amino acid at a highly conserved position, resulting in an amino acid substitution, p.V85E, predicted to be pathogenic by different tools. The substitution was located in the highly conserved HORMA domain of the protein (Supplemental Figure 2), known to mediate the REV7 interaction with REV1 and REV3 (10). Moreover, the absence of detectable levels of REV7 protein in several tissues, despite normal transcript levels, suggested that protein destabilization may result from the mutation (Figure $1 \mathrm{~F}$ ).

Lentiviral complementation of REV7 restores cellular and functional phenotype in patient cells. To provide additional evidence for the disease causality of the REV7 mutation, we lentivirally reexpressed a WT REV7 cDNA in patient fibroblasts and EBV-transformed cells. Western blot analysis of whole cell lysates revealed that REV7 expression was restored in REV7-transduced cells (Figure 2A). Reexpression of the WT REV7 fully rescued chromosome breakage, cell cycle arrest, and cell proliferation defects (Figure 2, B-D and Supple- mental Figure 3). Ser15 phosphorylation of p53 and phosphorylated $\gamma \mathrm{H} 2 \mathrm{ax}$ levels was also decreased in the REV7 complemented cells, and transcript analysis revealed a decrease of CDKN1A (Figure 2, A and E). Moreover, EGF123 cells exhibited an excess of $\gamma \mathrm{H} 2 \mathrm{AX}$ and 53BP1 foci, suggesting unresolved DNA damage at steady state and upon MMC exposure, and these defects were resolved by REV7 reexpression (Figure 2, A, F, and G, and Supplemental Figure 4).

CRISPR/Cas9-mediated knockout of REV7 recapitulates an FA phenotype. To confirm that loss of REV7 directly causes the FA phenotype, we used CRISPR/Cas9-mediated gene editing to generate a homozygous knockout in U2OS cells (denoted $R E V 7^{-1-}$ ) (Figure 3). Western blotting confirmed the absence of detectable REV7 protein in the $R E V 7^{-/-}$line, whereas REV7 was readily detectable in the parental cell line (Figure 3A). Consistent with the patient-derived EGF123 cells, FANCD2 monoubiquitination was largely unaffected in $R E V 7^{-/-}$cells. As expected, $R E V 7^{-/-}$cells exhibited the hallmark FA phenotypes, including an increase in chromosome breaks and radials upon MMC treatment, cellular hypersensitivity to MMC, and a pronounced $\mathrm{G}_{2} / \mathrm{M}$ arrest (Fig- 
A

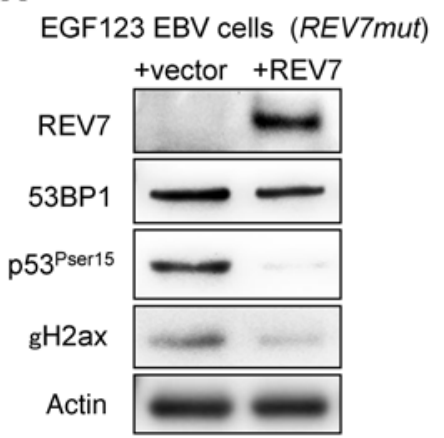

B

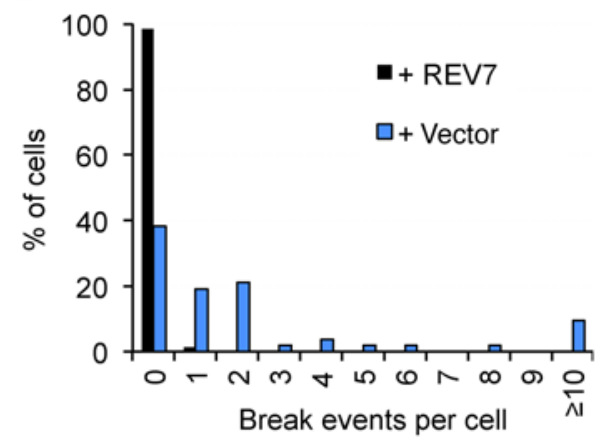

C

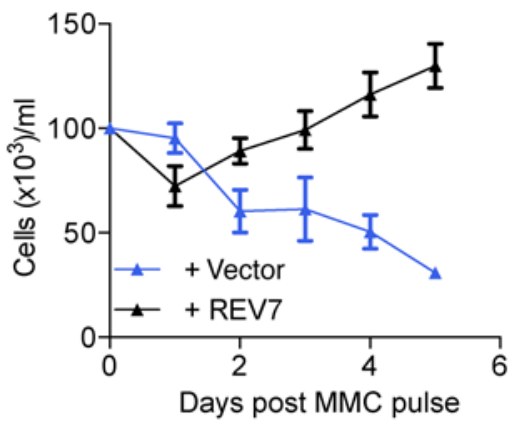

D

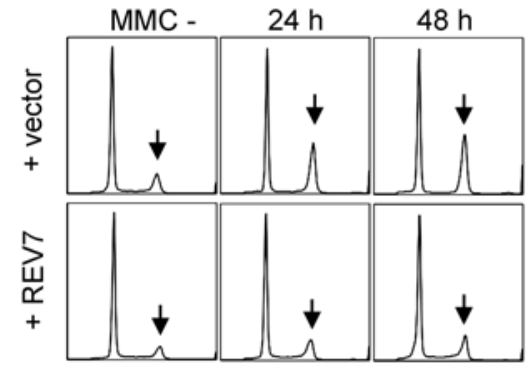

$\mathbf{F}$

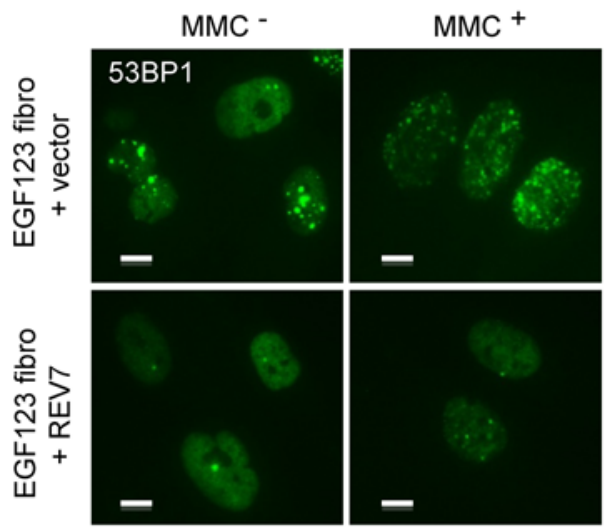

E

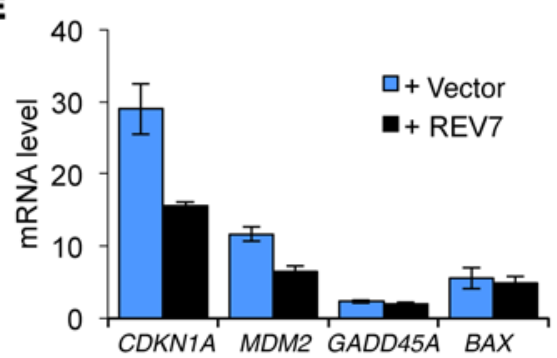

G

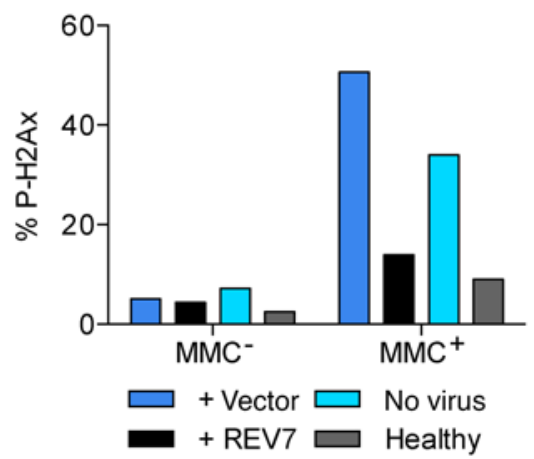

Figure 2. Correction of the extended phenotype of the EGF123 cells by WT REV7 expression. (A) Protein immunoblot analysis 24 hours after MMC pulse of EBV-transformed cells from the patient transduced with REV7 or the empty vector. (B) Quantification of MMC-induced breaks per cell. (C) Proliferation curves after MMC pulse. (D) Cell-cycle analysis after MMC pulse; arrows show late $S / G_{2}$ arrests. (E) Transcript expression levels of a set of DNA damage response genes analyzed by qRT-PCR relative to HPRT. (F) 53BP1 immunofluorescence analysis of ECF123 fibroblasts transduced with REV7 or empty vector, with or without MMC exposure. Scale bars: $10 \mu \mathrm{m}$. (G) Percentage of $\gamma \mathrm{H} 2 \mathrm{AX}$-positive cells by flow cytometry analysis with or without MMC exposure. Experiments shown in panels B-F were performed using EBV-transformed cells.

ure 3, C-F). All of these phenotypes were corrected by retroviral transduction with WT REV7 cDNA (Figure 3, B-F).

REV7 deficiency directly impairs hematopoietic cells. Previous studies have indicated that FA proteins are required for normal hematopoiesis and hematopoietic progenitor cell (HPC) survival $(6-8,18)$. To evaluate whether REV7 deficiency can affect hematopoiesis, we silenced this gene in mouse HPCs. Rev7 knockdown impaired the ability of HPCs to form in vitro CFU in short-term methylcellulose culture, similar to the defect observed in $\mathrm{Fancg}^{-/}$HPCs (Figure 3G). In addition, colonies had a decreased proportion of $\mathrm{c}-\mathrm{Kit}^{+} /$progenitor cells and an increased proportion of $\mathrm{CD}_{11} \mathrm{~b}^{+} /$differentiated cells (Figure 3H). Together, these data suggest that the Rev7 defect directly alters HPCs, leading to increased lineage engagement, consistent with a DNA damage-mediated mechanism of BMF.
FA cells are hypersensitive to DNA ICL agents and exhibit DNA repair and replication fork defects. How a deficiency in REV7 contributes to these cellular phenotypes remains unknown. Previous studies have demonstrated that the REV7 protein has several normal cellular functions. First, REV7 is a subunit of the DNA polymerase $\zeta$ complex (POL $\zeta$ ), a TLS error-prone polymerase complex containing REV1, REV7, REV3, and 2 additional accessory subunits, POLD2 and POLD3 $(9,10,19)$. POL $\zeta$ acts downstream of the FA core proteins in the repair of DNA ICL lesions $(3-5,20)$. In this role, REV7 acts as an adaptor protein between REV1 and REV3, the catalytic subunit of the POL $\zeta$ complex. A deficiency in REV7 could account for the well-known hypomutability of FA cells, resulting from a defect in TLS repair (20-23). Second, REV7 plays a role in mitosis by preventing premature activation of the 

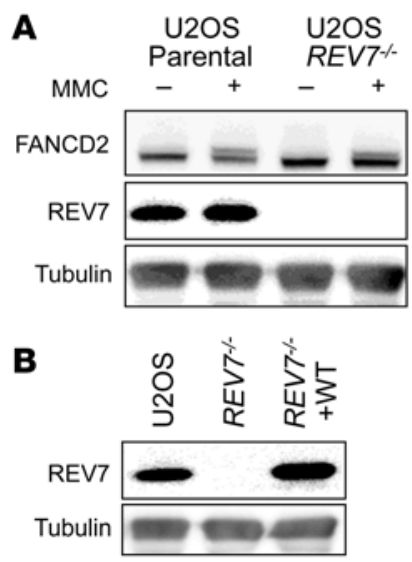
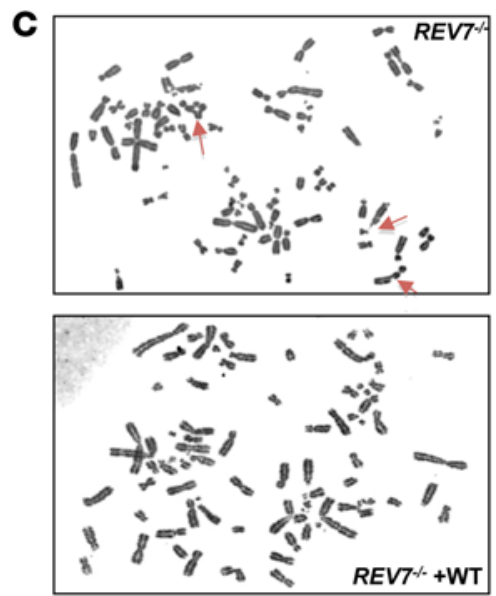
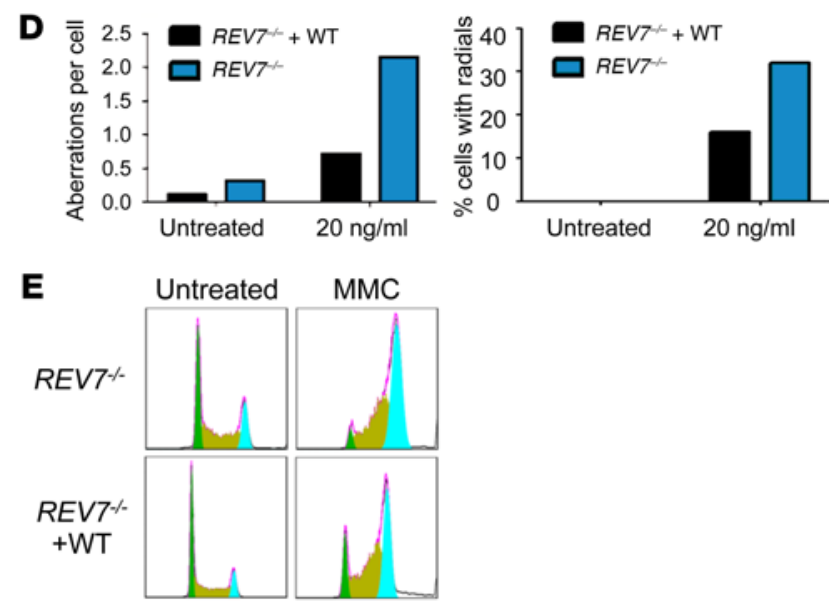

$\mathbf{F}$

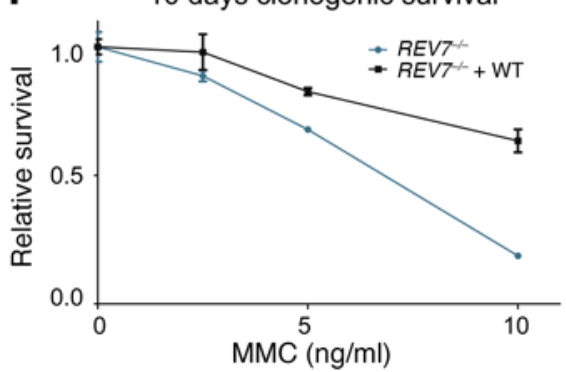

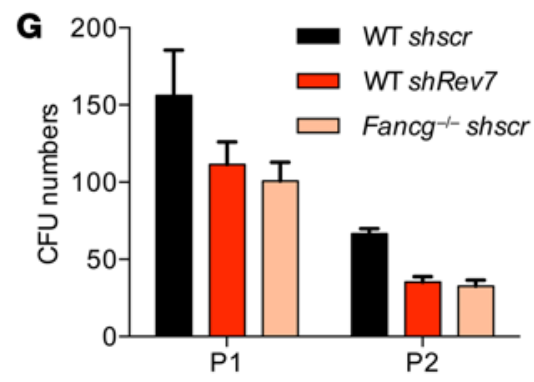

H

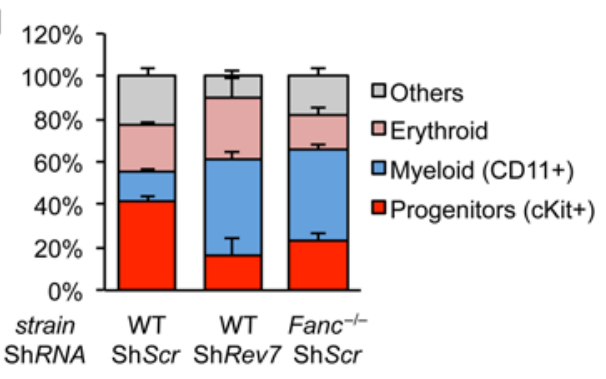

Figure 3. CRISPR/Cas9 knockout of REV7 recapitulates the FA phenotype. (A) Western blot of whole cell lysates shows no detectable REV7 protein in the REV7-1- line. FANCD2 is still efficiently monoubiquitylated in response to $100 \mathrm{ng} / \mathrm{ml} \mathrm{MMC.} \mathrm{(B)} \mathrm{Western} \mathrm{blot} \mathrm{showing} \mathrm{reconstitution} \mathrm{of} \mathrm{WT} \mathrm{REV7}$ expression in $R E V 7^{-1-}$ cells. (C-D) Increase in chromosome aberrations (left graph) and radials (right) after 48-hour treatment with $20 \mathrm{ng} / \mathrm{ml} \mathrm{MMC}$ as compared with cells complemented with WT REV7 cDNA. Original magnification, $\times 1,000$. (E) Increase in $\mathrm{G}_{2} / \mathrm{M}$ arrest with and without MMC treatment $(20 \mathrm{ng} / \mathrm{ml})$. (F) Impaired clonogenic capacity of REV7-/- cells over 10-day treatment with MMC. (G) REV7 is required for normal hematopoiesis; CFU scoring of bone marrow Lin ${ }^{-}$cells after lentiviral silencing using REV7 or scramble (scr) shRNAs. Fancg ${ }^{-1}$ cells are used as FA cell control. CFUs were numbered after 7 days on methylcellulose; 2 passages ( $\mathrm{P} 1$ and $\mathrm{P} 2$ ) were performed. (H) Cell population percentages in CFUs after 7 days of culture.

anaphase promoting complex/cyclosome $(\mathrm{APC} / \mathrm{C})$ by the activation of CDH1 $(11,12)$. Accordingly, a deficiency in REV7 could account for the mitotic defects and cytokinesis failure observed in FA cells (24-26). Third, REV7 participates in the cellular choice of DNA repair pathways during DNA double-strand break repair. In this role, REV7 functions downstream of 53BP1 and RIF1 and inhibits the 5' DNA end resection, thereby promoting nonhomologous end joining (NHEJ) and suppressing homologous recombination (HR) $(13,14)$. A deficiency in REV7 could therefore account, at least in part, for the dysregulated levels of NHEJ repair and HR repair observed in FA cells $(27,28)$.

Previous work in chicken DT40 cells had demonstrated that inactivation of Rev7 confers a cell hypersensitivity to ICL agents, suggesting that REV7 may be an FA gene (29). Interestingly, 2 Rev7 deficient mouse models have been generated, $R e v 7^{-1}$ and $R e v 7^{C 7 O R}$ mice $(30,31)$. In one model, Rev $7^{-1}$ mice exhibited growth retardation and a partial embryonic lethal phenotype, and those mice that survived to adulthood were infertile and showed germ cell aplasia in the testes and ovaries (30). In the second model, a missense mutation in Rev C C $^{\text {ORR }}$ disrupted Pol $\zeta$ assembly, thereby impairing mouse development and the repair of genotoxic agent-induced DNA lesions (31). $\operatorname{Rev} 7^{C 70 R}$ mutant cells also showed decreased proliferation, increased apoptosis, and arrest in the $\mathrm{S}$ phase with extensive $\gamma \mathrm{H} 2 \mathrm{AX}$ foci in nuclei, indicating accumulation of DNA damage (31). The Rev7deficient mice, therefore, have a very similar defect in development, embryonic lethality, infertility, and DNA damage accumulation, which is also observed in other $\mathrm{Fanc}^{-/}$mouse models (32).

In conclusion, these human genetic and functional data establish REV7 as a new bona fide FA gene, FANCV.

\section{Methods}

Further details can be found in the Supplemental Methods.

Samples and cell lines. This study was based on a cohort of 268 consecutive FA patients referred for medical diagnosis at the French Reference Center for Constitutional Bone Marrow Failure, Saint-Louis and Robert Debré Hospitals. All patients had an FA diagnosis based on FA tests, including the chromosomal breakage test. Primary fibroblasts were established and cells were analyzed at early passage. Genetic analyses of the FANC gene mutations were performed in fibroblast genomic DNA. EBV-transformed cell lines were produced for functional analyses (7).

Exome sequencing. All sequencing data were deposited in ArrayExpress (E-MTAB-4817).

CRISPR-Cas9 knockout. Guide RNA sequences were cloned into the pSpCas9(BB)-2A-GFP (PX458) vector, a gift from Feng Zhang (Addgene plasmid no. 48138; Massachusetts Institute of Technology, Cambridge, Massachusetts, USA.) . The genomic sequence targeted for 
CRISPR-Cas9 disruption in REV7 was GAGGTCTTGTCGTGTGAGCG. U2OS cells were transfected using Lipofectamine 2000 (Invitrogen catalog 11668). Twenty-four hours after transfection, $\mathrm{GFP}^{+}$cells were selected and single cells were seeded using a BD FACSAria II cell sorter. Single cells were grown for approximately 3 weeks. Putative REV7 knockouts were identified using Western blotting. For reconstitution, WT REV7 cDNA was cloned into the pBabe-puro retroviral vector.

Statistics. Results were evaluated by performing unpaired 2-tailed $t$ tests using GraphPadPrism version 5.0 software (GraphPad Software). Results are presented as mean \pm SEM.

Study approval. Informed consent for medical diagnosis and research was obtained from the patients and their relatives. The study was approved by the Institutional Review Board of Institut Universitaire d'Hématologie (IUH) at Saint-Louis Hospital (project IUH201212-078). All experiments in mice were performed in accordance with a protocol approved by the Committee on the Ethics of Animal Experiments Paris-Nord (C2EA-121), project No. 2014-IUH013.

\section{Author contributions}

DB, JMP, CC, AR, RC, CDE, WC, and SG performed experiments. All authors contributed to the research. DB, JMP, ADD, and JS designed the study and wrote the manuscript. All authors approved the final manuscript.

\section{Acknowledgments}

This work was supported by the European Research Council (ERC) Grant Consolidator 311660, an INCa grant (TRANSLA-12-011), and the Saint-Louis Institute program (ANR-10-IBHU-0002) (to JS). Saint-Louis/Robert Debré Hospital is supported by the French Government (Direction de l'Hospitalisation et de l'Organisation des Soins) as Centre de Référence Maladies Rares "Aplasies Médullaires Constitutionnelles" (to TL, GS, AB, JS). AR was supported by a fellowship from the Fondation ARC. ADD was supported by NIH grant DK43889. We thank the FA patients and their families, the Association Française de la Maladie de Fanconi (AFMF) for their support, and the physicians and nurses from French pediatric, genetic, and/or hematological centers who have taken care of the patients.

Address correspondence to: Jean Soulier, Saint-Louis Hospital and University Paris Diderot, Bâtiment Jean Bernard U944 INSERM, 1 Av. Claude Vellefaux, 75010 Paris, France. Phone: 33.15372.4041; E-mail: jean.soulier@sls.aphp.fr. Or to: Alan D. D'Andrea, Center for DNA Damage and Repair (CDDR), The Fuller-American Cancer Society Professor, Harvard Medical School, Department of Radiation Oncology, Dana-Farber Cancer Institute, Mayer 640, 450 Brookline Avenue, Boston, Massachusetts 02215, USA. Phone: 617.632.2112; E-mail: Alan_dandrea@dfci.harvard.edu.
1. Shimamura A, Alter BP. Pathophysiology and management of inherited bone marrow failure syndromes. Blood Rev. 2010;24(3):101-122.

2. Soulier J. Fanconi anemia. Hematology Am Soc Hematol Educ Program. 2011;2011:492-497.

3. Kottemann MC, Smogorzewska A. Fanconi anaemia and the repair of Watson and Crick DNA crosslinks. Nature. 2013;493(7432):356-363.

4. Bogliolo M, Surrallés J. Fanconi anemia: a model disease for studies on human genetics and advanced therapeutics. Curr Opin Genet Dev. 2015;33:32-40.

5. Ceccaldi R, Sarangi P, D'Andrea AD. The Fanconi anaemia pathway: new players and new functions. Nat Rev Mol Cell Biol. 2016;17(6):337-349.

6. Garaycoechea JI, Crossan GP, Langevin F, Daly M, Arends MJ, Patel KJ. Genotoxic consequences of endogenous aldehydes on mouse haematopoietic stem cell function. Nature. 2012;489(7417):571-575

7. Ceccaldi R, et al. Bone marrow failure in Fanconi anemia is triggered by an exacerbated p53/p21 DNA damage response that impairs hematopoietic stem and progenitor cells. Cell Stem Cell. 2012;11(1):36-49.

8. Walter D, et al. Exit from dormancy provokes DNA-damage-induced attrition in haematopoietic stem cells. Nature. 2015;520(7548):549-552.

9. Sharma S, Helchowski CM, Canman CE. The roles of DNA polymerase $\zeta$ and the Y family DNA polymerases in promoting or preventing genome instability. Mutat Res. 2013;743-744:97-110.

10. Tomida J, et al. REV7 is essential for DNA damage tolerance via two REV3L binding sites in mammalian DNA polymerase $\zeta$. Nucleic Acids Res. 2015;43(2):1000-1011.

11. Sale JE. REV7/MAD2L2: the multitasking maestro emerges as a barrier to recombination. EMBO J. 2015;34(12):1609-1611.

12. Listovsky T, Sale JE. Sequestration of CDH1 by MAD2L2 prevents premature APC/C activation prior to anaphase onset. J Cell Biol. 2013;203(1):87-100.

13. Xu G, et al. REV7 counteracts DNA double-strand break resection and affects PARP inhibition. Nature. 2015;521(7553):541-544.

14. Boersma V, et al. MAD2L2 controls DNA repair at telomeres and DNA breaks by inhibiting 5 ' end resection. Nature. 2015;521(7553):537-540.

15. Auerbach AD. Fanconi anemia diagnosis and the diepoxybutane (DEB) test. Exp Hematol. 1993;21(6):731-733.

16. Shimamura A, et al. A novel diagnostic screen for defects in the Fanconi anemia pathway. Blood. 2002;100(13):4649-4654.

17. Freie B, et al. Fanconi anemia type $\mathrm{C}$ and $\mathrm{p} 53$ cooperate in apoptosis and tumorigenesis. Blood. 2003;102(12):4146-4152.

18. Yung SK, et al. Brief report: human pluripotent stem cell models of fanconi anemia deficiency reveal an important role for fanconi anemia proteins in cellular reprogramming and survival of hematopoietic progenitors. Stem Cells. 2013;31(5):1022-1029.

19. Lee YS, Gregory MT, Yang W. Human Pol $\zeta$ purified with accessory subunits is active in translesion DNA synthesis and complements Pol $\eta$ in cisplatin bypass. Proc Natl Acad Sci US A . 2014;111(8):2954-2959.

20. Knipscheer $P$, et al. The Fanconi anemia pathway promotes replication-dependent DNA interstrand cross-link repair. Science. 2009;326(5960):1698-1701.

21. Kim H, Yang K, Dejsuphong D, D'Andrea AD. Regulation of Rev1 by the Fanconi anemia core complex. Nat Struct Mol Biol. 2012;19(2):164-170.

22. Mirchandani KD, McCaffrey RM, D’Andrea AD. The Fanconi anemia core complex is required for efficient point mutagenesis and Rev1 foci assembly. DNA Repair (Amst). 2008;7(6):902-911.
23. Papadopoulo D, Guillouf C, Mohrenweiser H, Moustacchi E. Hypomutability in Fanconi anemia cells is associated with increased deletion frequency at the HPRT locus. Proc Natl Acad Sci US A. 1990;87(21):8383-8387.

24. Chan KL, Palmai-Pallag T, Ying S, Hickson ID. Replication stress induces sister-chromatid bridging at fragile site loci in mitosis. Nat Cell Biol. 2009;11(6):753-760.

25. Naim V, Rosselli F. The FANC pathway and BLM collaborate during mitosis to prevent micronucleation and chromosome abnormalities. Nat Cell Biol. 2009;11(6):761-768.

26. Vinciguerra P, Godinho SA, Parmar K, Pellman D, D'Andrea AD. Cytokinesis failure occurs in Fanconi anemia pathway-deficient murine and human bone marrow hematopoietic cells. J Clin Invest. 2010;120(11):3834-3842.

27. Adamo A, et al. Preventing nonhomologous end joining suppresses DNA repair defects of Fanconi anemia. Mol Cell. 2010;39(1):25-35.

28. Pace P, Mosedale G, Hodskinson MR, Rosado IV, Sivasubramaniam M, Patel KJ. Ku70 corrupts DNA repair in the absence of the Fanconi anemia pathway. Science. 2010;329(5988):219-223.

29. Okada T, et al. Multiple roles of vertebrate REV genes in DNA repair and recombination. Mol Cell Biol. 2005;25(14):6103-6111.

30. Watanabe N, et al. The REV7 subunit of DNA polymerase $\zeta$ is essential for primordial germ cell maintenance in the mouse. J Biol Chem. 2013;288(15):10459-10471.

31. Khalaj M, et al. A missense mutation in Rev7 disrupts formation of Pol $\zeta$, impairing mouse development and repair of genotoxic agent-induced DNA lesions. J Biol Chem. 2014;289(6):3811-3824.

32. Parmar K, D’Andrea A, Niedernhofer LJ. Mouse models of Fanconi anemia. Mutat Res. 2009;668(1-2):133-140. 